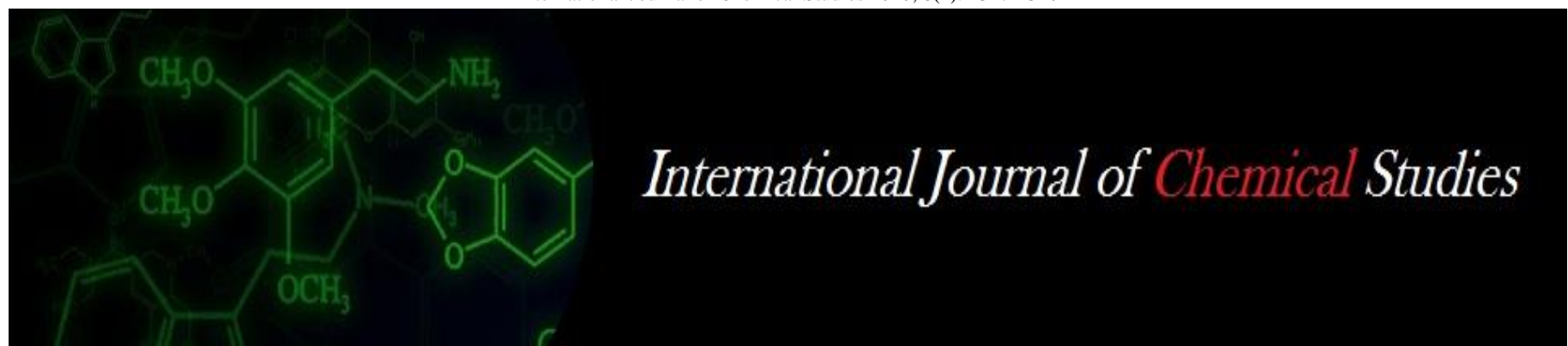

P-ISSN: 2349-8528

E-ISSN: 2321-4902

www.chemijournal.com

IJCS 2020; 8(2): 2317-2320

(C) 2020 IJCS

Received: 13-01-2020

Accepted: 15-02-2020

Amit Kumar Shukla

Department of horticulture

IGKV, Raipur, Chhattisgarh, India

Jitendra Singh

Department of horticulture IGKV, Raipur, Chhattisgarh, India

Corresponding Author: Amit Kumar Shukla Department of horticulture IGKV, Raipur, Chhattisgarh, India

\section{Studies on physico-chemical evaluation of tamarind (Tamarindus indica L.) genotypes prevailing in bastar region of Chhattisgarh on macro nutrient status of tamarind seed}

\author{
Amit Kumar Shukla and Jitendra Singh
}

DOI: https://doi.org/10.22271/chemi.2020.v8.i2ai.9094

\begin{abstract}
The present investigation entitled "Studies on physico-chemical evaluation of tamarind (Tamarindus indica L.) genotypes prevailing in Bastar region of Chhattisgarh" was carried out in the laboratory, Department of Horticulture, College of Agriculture, IGAU, Raipur (C.G.) during the year 2004-05 and 2005-06. The study was carried out with 16 treatments (genotypes) consist of ripe fruits collected from selected trees of tamarind exist in Tokapal and Jagdalpur block of Bastar district (C.G.) under Randomized Block Design with three replications.Higher Mg content of seed was noticed in IGTAM-15 $(39.25 \mathrm{mg} / 100 \mathrm{~g})$ and minimum was noticed in IGTAM-6 (14.58 mg/100 g).Maximum Cu content of seed was observed in IGTAM-15 $(16.14 \mathrm{mg} / 100 \mathrm{~g})$ and minimum content was observed in IGTAM-4 (1.79 $\mathrm{mg} / 100 \mathrm{~g})$. Higher Fe content of seed was recorded in IGTAM-15 $(6.13 \mathrm{mg} / 100 \mathrm{~g})$ minimum was recorded in IGTAM-5 (1.64 mg/100g). Maximum Ca content of seed was noticed in IGTAM-15 (557.53 mg/100g) and minimum content was noticed in IGTAM-7 $(45.03 \mathrm{mg} / 100 \mathrm{~g})$. Higher P content of seed was recorded in IGTAM-15 (142.74 mg/100g) and minimum status was recorded in IGTAM-5 (70.42 mg/100g).
\end{abstract}

Keywords: Tamarind, phosphorous, potassium, calcium and magnesium

\section{Introduction}

Tamarind (Tamarindus indica L.) is a hardy evergreen monotypic tree which belongs to the family 'Leguminosae' and sub-family Caesalpinaceae and has the chromosome number $2 n=24$. The name tamarind was derived from the Arabic word 'Tamar-E-Hind' meaning 'Date of India'. It is cultivated throughout the tropics and sub-tropics of the world and has become naturalized at many places.

Tamarind is an economically important tree of India as well as Chhattisgarh. In India, it is abundantly grown in Madhya Pradesh, Bihar, Andhra Pradesh, Tamil Nadu and Karnataka .

In India, tamarind is one of the most important common fruit trees and it is under cultivation for several centuries. Almost every part of it finds some use, but the most important is the fruit pulp which is the richest source of tartaric acid. It is being used in the manufacture of several products such as tamarind juice concentrate, pulp powder, pectin, pickle, chutneys, sauces, soups, jam, syrups, candy, tartaric acid, alcohol, refreshing tamarind drinks and tamarind kernel powder.

In India, few improved varieties of tamarind are in existence, like PKM-1 of Periyakulam, Pratisthan of Maharashtra and Urigam of Tamil Nadu (Geetha, 1995) ${ }^{[4]}$. Looking to the large area of tamarind either in forest or in homestead of tribal people.

\section{Materials and method}

Phosphorus

The total phosphorus was determined by using spectrophotometer (Olsen et al., 1954) and Vanado molybdate yellow reagent procedure. In this procedure $0.5 \mathrm{~g}$ sample was digested in $10 \mathrm{ml}$ diacid $\left(\mathrm{HNO}_{3}\right.$ and $\mathrm{HClO}_{4}$ 9:4) and then using yellow reagent (Ammonium molybdate + Ammonium meta vanadate + nitric acid) in aliquots. 


\section{Potassium}

Potassium $(\mathrm{K})$ and sodium $(\mathrm{Na})$ was determined by flame photometer method as described by Chapman and Pratt (1961).

\section{Calcium}

Calcium was determined by oxalatory method as described by Chapman (1928).

\section{Magnesium}

Magnesium was determined by thiazole yellow method as described by Young and Gill (1951).

\section{Results and Discussion \\ Phosphorus (seed)}

Data gathered on phosphorus (P) content of seed are presented in Table 1.

It is apparent from the data that phosphorus content of seed in different genotypes included in this study ranged between $70.46 \mathrm{mg} / 100 \mathrm{~g}$ (IGTAM-5) to $142.74 \mathrm{mg} / 100 \mathrm{~g}$ (IGTAM-15) during $1^{\text {st }}$ year $(2004-05), 70.42 \mathrm{mg} / 100 \mathrm{~g}$ (IGTAM-5) to $142.41 \mathrm{mg} / 100 \mathrm{~g}$ (IGTAM-15) during $2^{\text {nd }}$ year (2005-06) and $70.44 \mathrm{mg} / 100 \mathrm{~g}$ (IGTAM-5) to $142.58 \mathrm{mg} / 100 \mathrm{~g}$ (IGTAM-15) in case of pooled data (mean of both the years). Significant difference was observed among the genotypes in respect of phosphorus content of seed during $1^{\text {st }}$ year and $2^{\text {nd }}$ year of study as well as in pooled mean basis.

During $1^{\text {st }}$ year (2004-05), the highest phosphorus content of seed was noticed in IGTAM-15 (142.74 mg/100g), which was found significantly superior than all the genotypes studied in this investigation. This genotype (IGTAM-15) was followed by IGTAM-9 (131.20 mg/100g), IGTAM-16 (126.65 $\mathrm{mg} / 100 \mathrm{~g})$, and IGTAM-10 (122.48 mg/100g). The lowest phosphorus content of seed was observed in IGTAM-5 (70.46 $\mathrm{mg} / 100 \mathrm{~g}$ ).

During $2^{\text {nd }}$ year (2005-06), the maximum phosphorus content of seed was recorded in IGTAM-15 (142.41 mg/100g), which was found better than all the genotypes studied in this investigation. This genotype (IGTAM-15) was followed by IGTAM-9 (131.24 mg/100g), IGTAM-16 (126.46 mg/100g). The minimum phosphorus content of seed was noticed in IGTAM-5 (70.42 mg/100g).

In case of pooled data, the highest phosphorus content of seed was observed in IGTAM-15 (142.58 mg/100g), which was found excellent than all the genotypes studied in this investigation. This genotype was followed by IGTAM-9 (131.22 mg/100g), IGTAM-16 (126.56 mg/100g) and IGTAM-10 (122.56 mg/100g). The lowest phosphorus content of seed was recorded in IGTAM-5 (70.44 mg/100g).

Thus, the data obtained on phosphorus content of seed clearly reveal that maximum phosphorus content of seed was noticed in IGTAM-15 and minimum phosphorus content of seed was observed in IGTAM-5 in case of both the years as well as in pooled data.

In the present investigation, variations were also observed in calcium $(\mathrm{Ca})$ and phosphorus $(\mathrm{P})$ content of seed among the different tamarind genotypes studied (Table 1).

The phosphorus content of seed ranged between 70.42 $\mathrm{mg} / 100 \mathrm{~g}$ (IGTAM-5).The difference in mineral (Ca and P) content of tamarind pulp was possibly due to genetic difference among the genotypes studied in this investigation. These results are in close conformity with the finding of Ishola et al. (1990) ${ }^{[5]}$ and Bhattacharya et al. (1994) ${ }^{[2]}$, who reported that tamarind seed contain 68.40 to $165.00 \mathrm{mg} / 100 \mathrm{~g}$ phosphorus.
Table 1: Variation in mineral composition of different tamarind genotypes ( $\mathrm{P}$ content of seed)

\begin{tabular}{|c|c|c|c|}
\hline \multirow{2}{*}{ Treatments } & \multicolumn{3}{|c|}{ Phosphorus (mg/100 g) } \\
\cline { 2 - 4 } & $\mathbf{2 0 0 4 - 0 5}$ & $\mathbf{2 0 0 5 - 0 6}$ & Pooled \\
\hline IGTAM-1 & 95.50 & 95.14 & 95.32 \\
\hline IGTAM-2 & 92.36 & 92.17 & 92.26 \\
\hline IGTAM-3 & 111.72 & 112.55 & 112.14 \\
\hline IGTAM-4 & 74.77 & 74.38 & 74.58 \\
\hline IGTAM-5 & 70.46 & 70.42 & 70.44 \\
\hline IGTAM-6 & 74.44 & 74.12 & 74.28 \\
\hline IGTAM-7 & 97.71 & 98.58 & 98.14 \\
\hline IGTAM-8 & 115.92 & 116.06 & 115.99 \\
\hline IGTAM-9 & 131.20 & 131.24 & 131.22 \\
\hline IGTAM-10 & 122.43 & 122.38 & 122.51 \\
\hline IGTAM-11 & 108.67 & 108.54 & 108.60 \\
\hline IGTAM-12 & 80.63 & 80.30 & 80.47 \\
\hline IGTAM-13 & 76.28 & 76.23 & 76.26 \\
\hline IGTAM-14 & 94.23 & 94.05 & 94.14 \\
\hline IGTAM-15 & 142.74 & 142.41 & 142.58 \\
\hline IGTAM-16 & 126.65 & 126.46 & 126.56 \\
\hline SE(m) \pm & 0.2269 & 0.0918 & 0.1378 \\
\hline CD(5\%) & 0.66 & 0.27 & 0.40 \\
\hline
\end{tabular}

\section{Potassium (seed)}

The data recorded on potassium (K) content of seed are presented in Table 2.

A perusal of data indicate that potassium content of seed in different genotypes included in this study varied from 263.74 $\mathrm{mg} / 100 \mathrm{~g}$ (IGTAM-10) to $523.83 \mathrm{mg} / 100$ (IGTAM-15) during $1^{\text {st }}$ year (2004-05), $262.76 \mathrm{mg} / 100 \mathrm{~g}$ (IGTAM-10) to 523.42 $\mathrm{mg} / 100 \mathrm{~g}$ (IGTAM-15) during $2^{\text {nd }}$ year $(2005-06)$ and 263.25 $\mathrm{mg} / 100 \mathrm{~g}$ (IGTAM-10) to IGTAM-15 $(523.46 \mathrm{mg} / 100 \mathrm{~g})$ in case of pooled data (mean of both the years). Significant difference was observed among the genotypes in respect of potassium content of seed, during $1^{\text {st }}$ year and $2^{\text {nd }}$ year of study as well as pooled basis.

During $1^{\text {st }}$ year (2004-05), the maximum potassium content of seed was recorded in IGTAM-15 (523.83 mg/100g), which was found remarkably better than all the genotypes studied in this investigation. This genotype (IGTAM-15) was followed by IGTAM-16 (504.92 mg/100g), IGTAM-9 (412.60 $\mathrm{mg} / 100 \mathrm{~g})$. The minimum potassium content of pulp was recorded in IGTAM-10 $(263.74 \mathrm{mg} / 100 \mathrm{~g})$.

During $2^{\text {nd }}$ year (2005-06), the highest potassium content of pulp was noticed in IGTAM-15 (523.42 mg/100g), which was found remarkably better than all the genotypes included in this investigation. This genotype (IGTAM-15) was followed by IGTAM-16 (504.08 mg/100g), IGTAM-14 (413.24 $\mathrm{mg} / 100 \mathrm{~g})$ and IGTAM-9 (412.73 $\mathrm{mg} / 100 \mathrm{~g})$. The lowest potassium content of seed was observed in IGTAM-10 (262.76 mg/100g).

In case of pooled data, the maximum potassium content of pulp was recorded in IGTAM-15 (523.42 mg/100g), which was found remarkably better than all the genotypes studied in this investigation. This genotype (IGTAM-15) was followed by IGTAM-16 (504.50 $\mathrm{mg} / 100 \mathrm{~g}), \quad$ IGTAM-9 (412.67 $\mathrm{mg} / 100 \mathrm{~g})$ and IGTAM-14 (412.67 mg/100g). The minimum potassium content of seed was observed in IGTAM-10 (263.25 mg/100 g).

Thus, the data on potassium content of seed clearly revealed that highest potassium content of seed was observed in IGTAM-15 and lowest potassium content of seed was recorded in IGTAM-10 in case both the years as well as in pooled data. 
The maximum potassium content of seed was recorded in IGTAM-15 (523.83 mg/100g) and minimum potassium content of seed in IGTAM-10 (263.25 mg/100g), whereas.

The difference in mineral $(\mathrm{K})$ content of seed might be due to genetic nature of the genotypes. These results are supported by the findings of Ishola et al. (1990) [5], who reported that tamarind seed contain potassium 272.80 to $610.00 \mathrm{mg} / 100 \mathrm{~g}$ and sodium 19.20-28.80 mg/100g. Similar result on K and $\mathrm{Na}$ was also reported by Bhattacharya et al. (1994) ${ }^{[2]}$.

Table 2: Variation in mineral composition of different tamarind genotypes ( $\mathrm{K}$ content of seed)

\begin{tabular}{|c|c|c|c|}
\hline \multirow{2}{*}{ Treatments } & \multicolumn{3}{|c|}{ Potassium $(\mathbf{m g} / \mathbf{1 0 0}$ g) } \\
\cline { 2 - 4 } & $\mathbf{2 0 0 4 - 0 5}$ & $\mathbf{2 0 0 5 - 0 6}$ & Pooled \\
\hline IGTAM-1 & 320.33 & 320.70 & 320.52 \\
\hline IGTAM-2 & 270.23 & 270.53 & 270.38 \\
\hline IGTAM-3 & 275.12 & 275.21 & 275.17 \\
\hline IGTAM-4 & 285.25 & 285.10 & 285.18 \\
\hline IGTAM-5 & 300.12 & 300.79 & 300.45 \\
\hline IGTAM-6 & 290.31 & 290.21 & 290.26 \\
\hline IGTAM-7 & 300.24 & 300.25 & 300.21 \\
\hline IGTAM-8 & 400.13 & 400.87 & 400.50 \\
\hline IGTAM-9 & 412.60 & 412.73 & 412.67 \\
\hline IGTAM-10 & 263.74 & 262.76 & 263.25 \\
\hline IGTAM-11 & 280.51 & 280.21 & 280.36 \\
\hline IGTAM-12 & 301.88 & 301.41 & 301.65 \\
\hline IGTAM-13 & 319.82 & 320.31 & 320.06 \\
\hline IGTAM-14 & 412.09 & 413.24 & 412.67 \\
\hline IGTAM-15 & 523.83 & 523.42 & 523.46 \\
\hline IGTAM-16 & 504.92 & 504.08 & 504.50 \\
\hline SE(m) \pm & 0.2835 & 0.1949 & 0.1921 \\
\hline CD(5\%) & 0.82 & 0.56 & 0.55 \\
\hline
\end{tabular}

\section{Calcium}

Data recorded on calcium $(\mathrm{Ca})$ content of seed are presented in Table 3.

A perusal of data indicate that calcium content of seed in different genotype included in this study varied between $45.37 \mathrm{mg} / 100 \mathrm{~g}$ (IGTAM-7) to $557.5 \mathrm{mg} / 100 \mathrm{~g}$ (IGTAM-15) during $1^{\text {st }}$ year (2004-05), $45.03 \mathrm{mg} / 100 \mathrm{~g}$ (IGTAM-7) to $557.53 \mathrm{mg} / 100 \mathrm{~g}$ (IGTAM-15) during $2^{\text {nd }}$ year (2005-06) and $45.20 \mathrm{mg} / 100 \mathrm{~g}$ (IGTAM-7) to $557.58 \mathrm{mg} / 100 \mathrm{~g}$ (IGTAM-15) in case of pooled data (mean of both the years). Significant difference was observed among the genotypes in respect of calcium content of seed, during $1^{\text {st }}$ year and $2^{\text {nd }}$ year as well as in pooled basis.

During $1^{\text {st }}$ year (2004-05), the maximum calcium content of seed was noticed in IGTAM-15 $(557.5 \mathrm{mg} / 100 \mathrm{~g})$, which was found remarkably better than all the genotypes studied in this investigation. This genotype (IGTAM-15) was followed by IGTAM-8 (511.99 mg/100g), IGTAM-9 (426.4 mg/100g) and IGTAM-16 (931.67 mg/100g). The minimum calcium content of seed was observed in IGTAM-7 $(45.37 \mathrm{mg} / 100 \mathrm{~g})$.

During $2^{\text {nd }}$ year (2005-06), the highest calcium content of seed was recorded in IGTAM-15 (557.58 mg/100g), which was found excellent than all the genotypes studied in this investigation. This genotype (IGTAM-15) was followed by IGTAM-8 (512.17 mg/100g), IGTAM-9 (426.33 mg/100g) and IGTAM-16 (390.44 mg/100g). The lowest calcium content of seed was noticed in IGTAM-13 (95.14 mg/100g). In case of pooled data, the maximum calcium content of seed was observed in IGTAM-15 (557.52 mg/100g), which was found remarkably better than all the genotypes studied in this investigation. This genotype (IGTAM-15) was followed by IGTAM-8 (512.08 mg/100g), IGTAM-9 (426.38 mg/100g) and IGTAM-16 (391.05). The lowest calcium content of seed was noticed in IGTAM-7 (45.20 mg/100g).

Thus, the data recorded on calcium content of seed clearly show that highest calcium content of seed was noticed in IGTAM-15 and least calcium content in IGTAM-7 in case of both the years as well as in pooled data.

In the present investigation, variations were also observed in calcium $(\mathrm{Ca})$ and content of seed among the different tamarind genotypes studied (Table 2).

The maximum calcium content of seed was recorded in IGTAM-15 (557.53 mg/100g) and minimum calcium content of seed in IGTAM-7 $(45.03 \mathrm{mg} / 100 \mathrm{~g})$. While the phosphorus content of seed ranged between $70.42 \mathrm{mg} / 100 \mathrm{~g}$ (IGTAM-5) and $142.74 \mathrm{mg} / 100 \mathrm{~g}$ (IGTAM-15).

The difference in mineral $(\mathrm{Ca})$ content of tamarind pulp was possibly due to genetic difference among the genotypes studied in this investigation. These results are in close conformity with the finding of Ishola et al. (1990) ${ }^{[5]}$ and Bhattacharya et al. (1994) ${ }^{[2]}$, who reported that tamarind seed contain 9.30 to $786.00 \mathrm{mg} / 100 \mathrm{~g}$ calcium.

Table 3: Variation in mineral composition of different tamarind genotypes (Ca content of seed)

\begin{tabular}{|c|c|c|c|}
\hline \multirow{2}{*}{ Treatments } & \multicolumn{3}{|c|}{ Calcium (mg/100 g) } \\
\cline { 2 - 4 } & $\mathbf{2 0 0 4 - 0 5}$ & $\mathbf{2 0 0 5 - 0 6}$ & Pooled \\
\hline IGTAM-1 & 343.30 & 340.54 & 341.92 \\
\hline IGTAM-2 & 250.71 & 250.38 & 250.55 \\
\hline IGTAM-3 & 190.97 & 190.55 & 190.76 \\
\hline IGTAM-4 & 200.10 & 200.29 & 200.20 \\
\hline IGTAM-5 & 100.30 & 100.13 & 100.22 \\
\hline IGTAM-6 & 53.00 & 52.07 & 52.53 \\
\hline IGTAM-7 & 45.37 & 45.03 & 45.20 \\
\hline IGTAM-8 & 511.99 & 512.17 & 512.08 \\
\hline IGTAM-9 & 426.40 & 426.33 & 426.38 \\
\hline IGTAM-10 & 91.03 & 91.25 & 91.14 \\
\hline IGTAM-11 & 120.67 & 120.16 & 120.42 \\
\hline IGTAM-12 & 110.51 & 110.22 & 110.37 \\
\hline IGTAM-13 & 80.66 & 80.32 & 80.49 \\
\hline IGTAM-14 & 322.54 & 322.66 & 322.60 \\
\hline IGTAM-15 & 557.50 & 557.53 & 557.52 \\
\hline IGTAM-16 & 391.67 & 390.44 & 391.05 \\
\hline SE(m) \pm & 0.2132 & 0.0399 & 0.2132 \\
\hline CD(5\%) & 0.62 & 0.12 & 0.62 \\
\hline
\end{tabular}

\section{Magnesium (seed)}

Data obtained on magnesium $(\mathrm{Mg})$ content of seed are presented in Table 4.

It is evident from the data that magnesium content of seed in different genotypes included in this study varied from 14.84 $\mathrm{mg} / 100 \mathrm{~g}$ (IGTAM-6) to $39.25 \mathrm{mg} / 100 \mathrm{~g}$ (IGTAM-15) during $1^{\text {st }}$ year (2004-05), $14.58 \mathrm{mg} / 100 \mathrm{~g}$ (IGTAM-6) to 39.00 $\mathrm{mg} / 100 \mathrm{~g}$ (IGTAM-15) during $2^{\text {nd }}$ year (2005-06) and 14.73 $\mathrm{mg} / 100 \mathrm{~g}$ (IGTAM-6) to $39.13 \mathrm{mg} / 100 \mathrm{~g}$ (IGTAM-15) in case of pooled data (mean of both the years). Significant difference was observed among the genotypes in respect of magnesium content of seed during both the years as well as in pooled data.

During $1^{\text {st }}$ year (2004-05), the maximum magnesium content of seed was recorded in IGTAM-15 $(39.25 \mathrm{mg} / 100 \mathrm{~g})$ which was found significantly superior than all the genotypes studied in this investigation. This genotype (IGTAM-15) was followed by IGTAM-16 (35.95 mg/100g), IGTAM-9 (24.01 $\mathrm{mg} / 100 \mathrm{~g})$ and IGTAM-14 (23.95 mg/100g). The minimum magnesium content of seed was noticed in IGTAM-6 (14.58 $\mathrm{mg} / 100 \mathrm{~g}$ ) which was found significantly lower than all the genotypes studied in this investigation except IGTAM-7 
(15.03 mg/100g), IGTAM-2 (15.14 mg/100g) and IGTAM-1 (15.17 mg/100g).

During $2^{\text {nd }}$ year (2005-06), highest magnesium content of seed was recorded in IGTAM-15 (39.00 mg/100g), which was found significantly excellent than all the genotypes studied in this investigation. This genotype (IGTAM-15) was followed by IGTAM-16 (36.65 mg/100g), IGTAM-9 (24.54 mg/100g) and IGTAM-14 (23.29 mg/100g). The lowest magnesium content of seed was observed in IGTAM-6 $(14.58 \mathrm{mg} / 100 \mathrm{~g})$ which was significantly lower than all the genotypes studied in this investigation except IGTAM-2 (14.58 mg/100g).

In case of pooled data, maximum magnesium content of seed was recorded in IGTAM-15 (39.13 mg/100g), which was found significantly better than all the genotypes studied in this investigation. This genotype (IGTAM-15) was followed by IGTAM-16 (36.30 mg/100g), IGTAM-9 (24.28 mg/100g) and IGTAM-14 (23.62 mg/100g). The minimum magnesium content of seed was recorded in IGTAM-6 $(14.73 \mathrm{mg} / 100 \mathrm{~g})$ which was found significantly lower than all the genotypes except IGTAM-2 (15.08 mg/100g).

Thus, the data presented on magnesium content of seed clearly show that highest magnesium content of seed was observed in IGTAM-15 and least magnesium content of seed was recorded in IGTAM-6 in case of both the years as well as in pooled data.

In present investigation, variations were also observed in magnesium (Mg), content of seed among the different tamarind genotypes studied (Table 4).

The maximum magnesium content of seed was recorded in IGTAM-15 (39.25 mg/100g) and minimum magnesium content of seed in IGTAM-6 $(14.58 \mathrm{mg} / 100 \mathrm{~g})$.

The difference in mineral $(\mathrm{Mg})$ content of seeds may be due to genetic nature of the genotypes. The results are also in close agreement with the findings of Ishola et al. (1990) ${ }^{[5]}$, who reported that tamarind seed contain magnesium (17.50$118.30 \mathrm{mg} / 100 \mathrm{~g})$, Similar results on $\mathrm{Mg}$ content of tamarind seed was also reported by Bhattacharya et al. (1994) ${ }^{[2]}$.

Table 4: Variation in mineral composition of different Tamarind genotypes $(\mathrm{Mg}$, content of seed)

\begin{tabular}{|c|c|c|c|}
\hline \multirow{2}{*}{ Treatments } & \multicolumn{3}{|c|}{ Magnesium $(\mathbf{m g} / \mathbf{1 0 0} \mathbf{g})$} \\
\cline { 2 - 4 } & $\mathbf{2 0 0 4 - 0 5}$ & $\mathbf{2 0 0 5 - 0 6}$ & Pooled \\
\hline IGTAM-1 & 15.17 & 15.27 & 15.22 \\
\hline IGTAM-2 & 15.14 & 15.03 & 15.08 \\
\hline IGTAM-3 & 16.60 & 17.40 & 17.00 \\
\hline IGTAM-4 & 17.42 & 17.48 & 17.45 \\
\hline IGTAM-5 & 16.23 & 16.88 & 16.56 \\
\hline IGTAM-6 & 14.89 & 14.58 & 14.73 \\
\hline IGTAM-7 & 15.03 & 15.66 & 15.35 \\
\hline IGTAM-8 & 17.97 & 18.59 & 18.28 \\
\hline IGTAM-9 & 24.01 & 24.54 & 24.28 \\
\hline IGTAM-10 & 22.27 & 22.06 & 22.16 \\
\hline IGTAM-11 & 17.07 & 17.21 & 17.14 \\
\hline IGTAM-12 & 21.50 & 21.57 & 21.54 \\
\hline IGTAM-13 & 16.52 & 16.48 & 16.50 \\
\hline IGTAM-14 & 23.95 & 23.29 & 23.62 \\
\hline IGTAM-15 & 39.25 & 39.00 & 39.13 \\
\hline IGTAM-16 & 35.95 & 36.65 & 36.30 \\
\hline SE(m) \pm & 0.2347 & 0.1787 & 0.1475 \\
\hline CD(5\%) & 0.68 & 0.52 & 0.43 \\
\hline
\end{tabular}

\section{References}

1. Anonymous. The Wealth of India: Raw materials. Council of Scientific and Industrial Research, New Delhi, 2000.
2. Bhattacharya S, Bal S, Mukherjee RK, Bhattacharya S. Studies on the characteristics of some products from tamarind (Tamarindus indica) kernel. J Food Sci. Technol. 1994; 31(5):372-376.

3. Challapilli AP, Chimmad VP, Hulamani NC. Studies on correlation between some fruit characters in tamarind fruits. Karnataka J Agri. Sci. 1995; 8(1):114-115.

4. Geetha V. Urigam tamarind - A unique variety. Spice India. 1995; 8(8):8-9.

5. Ishola MM, Agbaji EB, Agbaji AS. A chemical study of Tamarindus indica fruit grown in Nigeria. J Sci., Food Agri. 1990; 5(1):141-143.

6. Karale AR, Wagh AP, Pawar BG, More TA. Association of fruit characters in tamarind. J Maharashtra Agri. Universities, 1999; 24(3):319-320.

7. Miller JC, Gafer AK. A study of the synthesis of carotene in the sweet potato plant and root. Proc. Amer. Soc., Hort. Sci. 1958; 71:388-390.

8. Parvez SS, Parvez MM, Fujii Y, Gemma H. Analysis of chemical components and oxygen radical absorbance capacity of Tamarindus indica L. Japanese J Trop. Agri. 2003; 47(4):243-249. 